\title{
How much does student perception of course attributes impact student motivation?
}

\section{Dr. Margot A Vigeant, Bucknell University}

Margot Vigeant is a professor of chemical engineering at Bucknell University. She earned her B.S. in chemical engineering from Cornell University, and her M.S. and Ph.D., also in chemical engineering, from the University of Virginia. Her primary research focus is on engineering pedagogy at the undergraduate level. She is particularly interested in the teaching and learning of concepts related to thermodynamics. She is also interested in active, collaborative, and problem-based learning, and in the ways hands-on activities such as making, technology, and games can be used to improve student engagement.

\section{Dr. Amy F. Golightly, Bucknell University}

Amy Golightly is an associate professor of education at Bucknell University. She earned her B. A. in psychology from the University of Saint Thomas, and her Ph.D. in school psychology from the University of Iowa. Her main research interests lie in understanding factors that facilitate or hinder learning and conceptual change in undergraduate students, and in development of assistive technology to help college students with disabilities. She is currently involved in collaborative research projects focused on these topics in chemical and electrical engineering. 


\section{How much does student perception of course attributes impact student motivation?}

Intrinsic motivation creates a more positive and engaged atmosphere in the classroom, and is positively correlated with students' persistence in engineering. While an instructor can't "intrinsically motivate" students, they certainly can create conditions that cultivate or defeat intrinsic motivation. In this study, the impact on students' motivational state of five course design features was measured using Guay, Vallerand, and Blanchard's Situational Motivation Scale (SIMS) (1). Course features considered included the incorporation of open-ended problem solving, physical realization of a design, incorporation of broad perspectives, interdisciplinary student teams, and "real" problems. These course features were aligned with Self-Determination Theory to create the conditions for enhanced student intrinsic motivation (2). Prior work suggested that intrinsic motivation was especially cultivated by having students work in an interdisciplinary environment, on problems for external clients or that were personally meaningful (7). However, this prior work only considered faculty-reported presence or absence of course design features. Surveys suggested that students and faculty were not in perfect agreement about the presence of certain course features, notably interdisciplinary-interactions and the extent to which problems were "real" and reflective of what students expect to see in their career or find personally meaningful. This study focuses on the students' perceptions of course elements and the extent to which students' perception of the presence or absence of these elements impacts their motivational state in their coursework.

\section{Introduction}

Intrinsic motivation, defined as the "inclination toward assimilation, mastery, spontaneous interest, and exploration"(2), is positively correlated with task-persistence and overall satisfaction with a given task (3). Because intrinsic motivation is a self-generated state, one might think that if faculty desire this as an outcome, they might have little impact on its presence or absence in a given student - that's what intrinsic means, after all. While faculty cannot truly "intrinsically motivate" students, they can create conditions where intrinsic motivation is more or less likely. For example, heavy emphasis on grades as a reward or punishment may drive students towards extrinsic motivation and away from intrinsic motivation (4). Students may be pushed towards amotivation when they don't understand why they are required to study a given topic (5).

According to Self Determination Theory (2) there are four main motivational states: intrinsic motivation, identified regulation, extrinsic motivation and amotivation. According to Guay et al., these motivational states are not mutually exclusive. Intrinsic motivation is the desire to engage in an activity for its own sake, because it is inherently interesting or enjoyable. Extrinsic motivation is generated by desire to attain some type of external reward, such as grades. Identified regulation is fostered when a person sees what they are doing as relevant to their future 
career, and is a form of extrinsic motivation. Amotivation is present when a person does not know why they are engaging in an activity or behavior.

A group of experienced instructors at our institution was seeking ways to improve their engineering classes to foster greater intrinsic motivation among students. Inspired by Ryan and Deci's Self-Determination Theory and their own prior experience, they hypothesized six course attributes that would be likely to contribute to students' intrinsic motivation. Table 1 shows the thematic grouping of these attributes according to Ryan and Deci's (2000) self-determination theory under the auspices of autonomy, competence and relatedness $(2,6)$. The faculty were seeking ways in which students might experience autonomy, competence, and relatedness within the context of including more design projects in courses. In this and our previous study, motivation and curiosity were hypothesized to be fostered when students had some choice in the learning activities (autonomy), they were challenged at an appropriate level (competence), and their work allowed them to interact with their peers and members of the community in meaningful ways (relatedness)(6).

Table 1: Course Attributes

Self-Determination Theory Category

Course Attribute

\{Ryan and Deci, 2000, \#79467\}

I. Autonomy

A. Course is voluntary

1. Students wanted to take the course

2. Course was not required for the student's major

B. Student work is informed by broad perspectives

C. Problems have multiple possible solutions and solution paths

II. Competence

A. In their coursework, students create a product of some sort

III. Relatedness

A. Students work with students from a discipline other than their own (interdisciplinary)

B. Students work on real problems

1. The work was important to a client

2. The work was similar to what students expect to see in their $\underline{\text { career }}$

3. The work was personally meaningful to students

Course attributes I.A and III.B were further subdivided for clarity. Faculty initially conceived of student's coursework as either required for their major or an elective (voluntary) course. However, depending on the lens through which one perceives the students, their choice of major was also a voluntary action. Therefore I.A was broken into the two sub-sections shown. III.B was asked independently and also further subdivided into three possible interpretations of what could make a problem "realistic" in the eyes of students.

Note that Table 1 should not be understood to imply that the only path towards students' experience of autonomy, competence, or relatedness is through these attributes. Rather, the goal 
is to determine if deliberate inclusion of these attributes, which are outside of the baseline requirements of our typical engineering courses, leads to greater student intrinsic motivation.

In our prior work (7), students' motivational state and curiosity was correlated with faculty's report of the presence or absence of the above attributes for the first academic year of data. However, it was noticed in reviewing results that faculty and students did not completely agree about the presence or absence of course attributes. Because we hypothesize that student attitude is most closely correlated with student perceptions of course design more than faculty's, the present study reexamines these data from the student perspective and includes an additional semester of data.

\section{Methods}

The present study builds upon prior work $(8,9)$ that used a multiple-choice self-report instrument to assess and compare motivation and curiosity in students who were taking elective courses, and the work of (7) that used the same instrument to examine whether certain course attributes positively affected students' entrepreneurial mindsets within the KEEN framework (10). This instrument has two components: The Situational Intrinsic Motivation Scale (SIMS; (1)), which was used to assess situational motivation, and a situational interest sub-scale by (11), which was used to assess the curiosity of engineering undergraduates. Both of these scales were previously validated by their respective authors. The present study uses only the SIMS component of the survey. The instrument is shown in Appendix A.

Student participation in this study was voluntary and it was granted 'exempt' status by the IRB. Participants' responses were confidential and aggregate student response to surveys was not shared with the faculty until after the semester's grades were finalized. Data were collected within two weeks of the start, midterm and end of the semester. Participants were sent an email with a link to the SIMS/Curiosity survey and asked to complete it while reflecting on the most recent instance of their course. Additionally, students were asked to complete a demographic questionnaire and a follow-up survey that asked whether the attributes described above were present or not in the course. Students were offered $\$ 10$ per completed survey; maximum compensation per student was therefore $\$ 50$.

A sample of convenience was used in this study. Undergraduate engineering majors in 13 courses were asked to participate. These courses were offered across the Fa 17, Sp 18, Fa 18 semesters. Students were typically $3^{\text {rd }}$ and $4^{\text {th }}$ year students and courses were offered by faculty in Electrical, Chemical, and Mechanical engineering departments, and were a mix of required core courses and upper-level technical elective courses. Chemical engineering Heat and Mass Transfer, Thermodynamics, and Design were all included in the study. Enrollments did not exceed 35 students. The courses were deliberately selected because the faculty believed at least one of the aforementioned attributes to be present to some degree. Therefore, it is likely that these attributes manifested somewhat differently across courses, and this variability is reflective 
of the realities of teaching. To preserve confidentiality, courses are not named with the results; rather, they are discussed in aggregate according to the relevant attributes.

SIMS/Curiosity responses from the third administration of the survey were used in this study (n =189). Subscale scores were obtained for intrinsic motivation, identified regulation, external motivation, and amotivation. Following Ryan and Deci (2), answers corresponding to "identified regulation" and "extrinsic motivation" were grouped into "extrinsic motivation" for this present study, as both express some degree of extrinsic motivation.

For the course attributes survey, responses were collected using a 5-point Likert-type scale, but for the purposes of this study, they were dichotomized from 'strongly agree' and 'agree' to 'yes' and 'strongly disagree' and 'disagree' into 'no'. Neutral answers were omitted from the analysis. Mean subscale scores were computed and compared between groups. Significance for differences between these groups was tested using oneway analyses of variance (ANOVA). Effect size estimates were computed using partial eta squared. SPSS was used for all calculations.

\section{Results and Discussion}

Table 2 summarizes the significance of all results from this study. Cells containing no data showed no significant change between that particular attribute's presence or absence. Three course attributes have significant and consistent impact across multiple types of motivation - I.A. (and its derivatives), the elective nature of the course, III.A., the extent to which students connect with others outside their own major (interdisciplinary), and III.B. (and its derivatives), the extent to which students feel they worked on "real" problems.

In the Ryan and Deci Self-Determination Theory (2) framework for intrinsic motivation, it's expected students will exhibit greater levels of intrinsic motivation and lower levels of amotivation when they can express autonomy, competence, and connection. As shown in Table 1 , the course attributes examined were intended to express these three elements. In discussing this study with colleagues, many anticipated that the most important attribute for motivation would be whether or not a course was an elective. While this is a very clear expression of autonomy, it was hypothesized that a novelty effect associated with course choice would exert less of an influence as the semester progressed; the data suggest that this was not the case. While attribute I.B., Broad Perspectives, did not show an effect, this may be more a factor of the lopsidedness of the data than an actual lack of an effect - 116 students agreed that they were encouraged to employ broad perspectives, while only 20 disagreed. A skewed sample likewise makes strong interpretation of attribute I.C. Multiple Solutions challenging.

The one course attribute aligned to competence, II.A. Creation of a Product, shows no impact on motivational state. A preliminary study (8) suggested that this course attribute was meaningful, 
but this has not held up in subsequent study. One reason may be that, for some students, building an actual prototype fails to enhance their feeling of competence. It requires more than calculation to create a functional prototype, and many students enter engineering programs without significant prior experience in building things. Actually creating a prototype requires iteration and struggle, and it's been shown similar cognitive effort is read as a sense of incompetence, even when they are demonstrably learning the material (12).

The course attributes mapped to connectedness most consistently demonstrate impact on students' motivational state. While creating a product was not important for motivation, the perceived "reality" of problems was consistently important - increasing both intrinsic and extrinsic motivation and decreasing amotivation. Faculty hypothesized that there were three attributes that might make a problem "real" for students (course attributes III.B.1-3). Being meaningful to a client, similar to imagined career, and/or personally meaningful all increased intrinsic motivation. 
Table 2: Results; any blank cell showed no statistically significant impact. Dark green shading indicates positive medium effect size, light green a positive small effect size, light pink a small negative effect size, and darker pink a negative medium effect size.

\begin{tabular}{|c|c|c|c|}
\hline Course Attribute & Intrinsic Motivation & Extrinsic Motivation & Amotivation \\
\hline I.A. Voluntary & $\begin{array}{l}\text { Significantly Higher, } \\
\text { Large effect size for } \\
\text { classes students } \\
\text { "wanted to take" } \\
\mathrm{p}<0.000 \eta^{2}=0.294 \\
\text { ALSO } \\
\text { Significantly Higher, } \\
\text { Small effect size for } \\
\text { classes that fulfill } \\
\text { requirements } \\
p<0.007 \eta^{2}=0.045\end{array}$ & $\begin{array}{l}\text { Significantly Higher, } \\
\text { Medium Effect size for } \\
\text { classes that fulfill } \\
\text { requirements } \\
p<0.001 \eta^{2}=0.072\end{array}$ & $\begin{array}{l}\text { Significantly Lower, } \\
\text { Medium effect size for } \\
\text { classes students } \\
\text { "wanted to take" } \\
p<0.000 \eta^{2}=0.132\end{array}$ \\
\hline \multicolumn{4}{|l|}{$\begin{array}{l}\text { I.B. Broad } \\
\text { perspectives }\end{array}$} \\
\hline \multicolumn{4}{|l|}{ I. C. Multiple solutions } \\
\hline \multicolumn{4}{|l|}{ II.A. Create a product } \\
\hline III.A. Interdisciplinary & $\begin{array}{l}\text { Significantly Higher, } \\
\text { Medium effect size } \\
p<0.000 \eta^{2}=0.082\end{array}$ & $\begin{array}{l}\text { Significantly Lower, } \\
\text { Small effect size } \\
p<0.031 \eta^{2}=0.031\end{array}$ & \\
\hline III.B. Real problems & $\begin{array}{l}\text { Significantly Higher, } \\
\text { Medium effect size } \\
p<0.000 \eta^{2}=0.077\end{array}$ & $\begin{array}{l}\text { Significantly Higher, } \\
\text { Small effect size } \\
p<0.017 \eta^{2}=0.036\end{array}$ & $\begin{array}{l}\text { Significantly Lower, } \\
\text { Medium effect size } \\
p<0.000 \eta^{2}=0.134\end{array}$ \\
\hline III.B.1. Client & $\begin{array}{l}\text { Significantly Higher, } \\
\text { Medium effect size } \\
p<0.002 \eta^{2}=0.070\end{array}$ & & \\
\hline III.B.2. Career & $\begin{array}{l}\text { Significantly Higher, } \\
\text { Small effect size } \\
p<0.004 \eta^{2}=0.064\end{array}$ & & $\begin{array}{l}\text { Significantly Lower, } \\
\text { Medium effect size } \\
p<0.000 \eta^{2}=0.111\end{array}$ \\
\hline $\begin{array}{l}\text { III.B.3. Personally } \\
\text { meaningful }\end{array}$ & $\begin{array}{l}\text { Significantly Higher, } \\
\text { Medium effect size } \\
p<0.000 \eta^{2}=0.122\end{array}$ & & $\begin{array}{l}\text { Significantly Lower, } \\
\text { Medium effect size } \\
p<0.001 \eta^{2}=0.090\end{array}$ \\
\hline
\end{tabular}

Comparing these results to prior work, while the details differ, the outcomes are the same (7). That is, the course attributes that appeared to significantly impact motivation in the earlier study continue to do so here. 


\section{Implications for course design}

To revisit the motivating question for this work: what can we as faculty and course designers $d o$ to create the conditions for intrinsic motivation in our courses? These results suggest several potential elements for design of a successful course.

While often outside of faculty control, we should be mindful of whether our course is required or fulfills some university requirement and if students want to take the course. Our results suggest that are electives and/or courses students want to take give a motivational boost that lasts all semester.

More critically for course design, these results suggest that the largest impact for the smallest change comes from the incorporation of "real" problems in the course. These problems could be "real" in any of the ways explored - personally meaningful, for a client, or similar to those students foresee in industry. Note that all three elements of "Real Problems" need not be present, our results suggest any one of them will do. This is helpful because while external client projects are effective, they require additional time, coordination, and intellectual property management that is not consistently available for all courses. However, finding problems that are related to future careers or are personally meaningful for students should be possible for all courses. Faculty can bring in stories from alumni or local AIChE sections to illuminate how current class topics are aligned with students' future careers. While it is harder for faculty to explicitly make class projects personally meaningful for students, it's possible through giving students reflection prompts to encourage student thinking along these lines (for example:(13)).

These results also suggest that broadening the disciplines of the students in the course will yield motivational benefits, in addition to the benefits of students having a broader technical base on which to draw for problem solving. Some students' reported that they engaged with students from a major other than their own in classes where the faculty member said all class enrollment was from a single major. We believe students' broader interpretation of Table 1's "interdisciplinarity" comes from their actions outside of class - for example, consulting with friends in other majors about the project, or being assigned to "talk to X potential customers". Faculty may therefore realize some of the benefits of an interdisciplinary course even when their course is restricted to a single major by encouraging or assigning students to interact more broadly on an assignment.

Class atmosphere and student learning both benefit when there are few amotivated students and a greater sense of intrinsic motivation in the classroom. While faculty cannot "intrinsically motivate" anyone but themselves, by incorporating interdisciplinary perspectives and real problems in their classes, they may be able to create the conditions where students are most likely to find their own intrinsic motivation. Two examples of pedagogical practice that might facilitate increased motivation could be offering students a choice from among multiple problem scenarios that accomplish the same pedagogical goal, or to have all students work on the most 
popular choice. Teaching an elective course is also helpful, but it is by no means the only way to encourage positive motivational states in students.

\section{Acknowledgement}

This work was conducted with support from the Kern Engineering Education Network (KEEN).

\section{References}

1. Guay, F., R. J. Vallerand, and C. Blanchard. 2000. On the assessment of situational intrinsic and extrinsic motivation: The Situational Motivation Scale (SIMS). Motivation and emotion 24: 175-213.

2. Ryan, R. M., and E. L. Deci. 2000. Self-determination theory and the facilitation of intrinsic motivation, social development, and well-being. American psychologist 55: 68.

3. Gottfried, A. E. 1985. Academic intrinsic motivation in elementary and junior high school students. Journal of educational psychology 77: 631.

4. Butler, R., and N. Mordecai. 1986. Effects of no feedback, task-related comments, and grades on intrinsic motivation and performance. Journal of Educational Psychology 78: 210-216.

5. Leroy, N., and P. Bressoux. 2016. Does amotivation matter more than motivation in predicting mathematics learning gains? A longitudinal study of sixth-grade students in France. Contemporary Educational Psychology 44-45: 41-53.

6. Vigeant, M., D. Silverstein, K. Dahm, L. Ford, J. Cole, and L. Landherr. 2018. How we teach: Unit Operations Laboratory. Proceedings of American Association for Engineering Education.

7. Vigeant, M., M. Prince, K. Nottis, and A. Golightly. 2018. Curious about student curiosity: Implications of pedagogical approach for students' mindset. Proceedings of American Association for Engineering Education.

8. Kim, C., R. A. Cheville, K. Nottis, J. Tranquillo, E. J. Jablonski, M. Prince, and M. Vigeant. 2015. Assessing situational curiosity and motivation in open-ended design electives. Proceedings of Frontiers in Education.

9. Nottis, K., M. Prince, M. Vigeant, C. Kim, and E. J. Jablonski. 2016. The effect of course type on engineering undergraduates' situational motivation and curiosity. Proceedings of American Society for Engineering Education.

10. KEEN, 2015. Entrepreneurial Mindset Framework. KEENZiene

11. Chen, A., P. W. Darst, and R. P. Pangrazi. 1999. What constitutes situational interest? Validating a construct in physical education. Measurement in Physical Education and Exercise Science 3: 157-180.

12. Deslauriers, L., L. S. McCarty, K. Miller, K. Callaghan, and G. Kestin. 2019. Measuring actual learning versus feeling of learning in response to being actively engaged in the classroom. Proceedings of the National Academy of Science U S A 116: 19251-19257.

13. Tranquillo, J. 2016. Mirror mirror: Reflection and the building of mindsets. Proceedings of American Society for Engineering Education. 
Appendix A: Motivation Survey Instrument

Missing questions are repeats of "Q3" below, spaced to provide a reminder of the task.

Questions 4-22 are from SIMS (1), while 23-28 are from (11).

Q3. The questions on this page will ask you about activities you did in your most recent class meeting for the class indicated above.

Q4.

Why are you (were you) engaged in activities related to your most recent class?

Q5. Because I think that this activity is interesting.

Q6. Because I am doing it for my own good.

Q7. Because I am supposed to do it.

Q8. There may be good reasons to do this activity, but personally I don't see any.

Q9. Because I think that this activity is pleasant.

Q10. Because I think this activity is good for me.

Q12. Because it is something I have to do.

Q13. I do this activity, but I am not sure it is worth it.

Q14. Because this activity is fun.

Q15. By personal decision.

Q16. Because I don't have any choice.

Q17. I don't know; I don't see what this activity brings me.

Q18. Why are you (were you) engaged in activities related to your most recent class? 
Q19. Because I feel good when I do this activity.

Q20. Because I believe this activity is important for me.

Q21. Because I feel I have to do it.

Q22. I do this activity, but I am not sure it is a good thing to pursue it.

Q23. Because this activity is fascinating.

Q25. I want to discover all the tricks in this activity.

Q26. I like to find out more about how to do this activity.

Q27. I like to inquire into the details of how to do this activity.

Q28. Because I want to analyze it to have a grip on this activity. 